\title{
Ist die Wirkung von Lean Hospital auf der Bettenstation messbar?
}

\author{
Heiko Behrendt ${ }^{a, e}$, Sarah Niederberger ${ }^{b, e}$, Gioia Braunc, Katharina Rüther-Wolfd,e \\ ${ }^{a}$ Dr., Experte für Patientenzentriertes Management; ${ }^{b}$ M.A., Expertin für Patientenzentriertes Management ${ }^{c}$ Assistenzärztin, Reha Rheinfelden; ${ }^{d}$ Dr. med., \\ MBA, Fachärztin für Gynäkologie und Geburtshilfe, Leiterin Abteilung Patientenzentriertes Management; ${ }^{e}$ Ärztliche Direktion, Universitätsspital Basel
}

\begin{abstract}
Zahlreiche Schweizer Spitäler wenden Lean Hospital in Form von Programmen und Projekten an. Der steigende Druck zur Kostenreduktion im Gesundheitswesen bedingt, dass die Wirksamkeit von Lean Hospital belegt wird. Ausgehend vom Grundproblem des Erkennens und Zuordnens der Wirkungen in einem komplexen Umfeld wurde ein Modell entwickelt, welches die Wirkungen für eine Lean Bettenstation darstellt.
\end{abstract}

\begin{abstract}
Die Einführung des SwissDRG-Systems fördert den Innovations- und Qualitätswettbewerb, da aufgrund fester Abgeltungen eine Kosten- und Preisführerschaft erschwert, bzw. nicht mehr möglich ist. Transparenz wird sowohl vom Bund gefordert als auch von Vergleichsportalen und Rankings geliefert. Andere Branchen, wie beispielsweise die Automobilindustrie, standen schon früher vor ähnlichen Herausforderungen. Früh fiel Toyota auf: Sie dachten vom Kunden aus und waren mit einem effizienten Produktionssystem in der Lage, hohe Qualität und Produktivität zu liefern. Dieses System, Ende der 1980er Jahre von westlichen Wissenschaftlern und Forschern «Lean» getauft, verbrei-
\end{abstract}

\section{Résumé}

Depuis quelques années, les hôpitaux suisses tels que I'USB appliquent le Lean Hospital sous forme de programmes et de projets. Généralement, ceux-ci doivent, à juste titre, faire la preuve de leur efficacité au niveau interne. L'USB a donc mis au point un modèle des effets et l'a testé sur la base de l'exemple de deux services hospitaliers. Cet article montre comment les effets du Lean Hospital Management peuvent être identifiés et quantifiés et à quoi il faut être attentif. L'un des problèmes fondamentaux était d'identifier et d'attribuer les effets dans un contexte soumis à de nombreuses autres influences. Le modèle montre comment les outils du Lean Hospital utilisés se traduisent en réalisations, résultats et impact. Des indicateurs ont été développés pour les principaux éléments du modèle des effets et leur nombre a été systématiquement réduit. Le défi était de proposer des indicateurs équitables et mesurables et de veiller à ce qu'une éventuelle stratégie dérivée de l'évaluation soit fructueuse. En prenant pour exemple la durée de séjour, l'article montre comment un effet du Lean Hospital Management peut être identifié et évalué dans un service hospitalier. II expose en outre deux initiatives médicales qui remplissent d'importants critères Lean et ont également réduit la durée de séjour. tete sich zunächst in der Fertigungsindustrie, später auch in anderen Branchen [1]. Einige namhafte Unternehmen schafften mittels Lean Management den qualitativen und finanziellen Turn around [2]. Ende der 1990er Jahre entdeckten erste Spitäler Lean und wendeten die Prinzipien erfolgreich an [3]. Das Universitätsspital Basel (USB) startete 2014 ein Programm als Baustein zur Umsetzung der Spitalstrategie.

\section{Evaluation von Programmen}

Werden Programme in einem komplexen Umfeld wie einem Spital durchgeführt, ist es oft schwer, die gewünschte Wirkung zumessen bzw. nachzuweisen, da viele programminterne und -externe Einflussfaktoren gleichzeitig wirken [4]. Programme basieren zudem immer auf impliziten Annahmen über ihre Wirkung, die teilweise nicht mit der Realität übereinstimmen [5, 6]. In der Evaluation von Programmen sollten daher die zugrundeliegenden Wirkungsannahmen transparent dargestellt werden. Wirkungsmodelle können dies. Sie unterstützen die Beurteilung, in welchem Ausmass die identifizierten Effekte dem Programm und nicht einer anderen Ursache zugewiesen werden können. Auch projektexterne Einflüsse können identifiziert und deren Wirkung eingeschätzt werden [7].

\section{Wirkungsmodell einer Lean Bettenstation}

Da kein validiertes Kennzahlenset für die Bewertung der Performance einer Bettenstation vorliegt, wurde zunächst ein Wirkungsmodell erarbeitet, das die Wirkungen von Lean Hospital darstellt (vgl. Abb. 1). Daraus wur- 


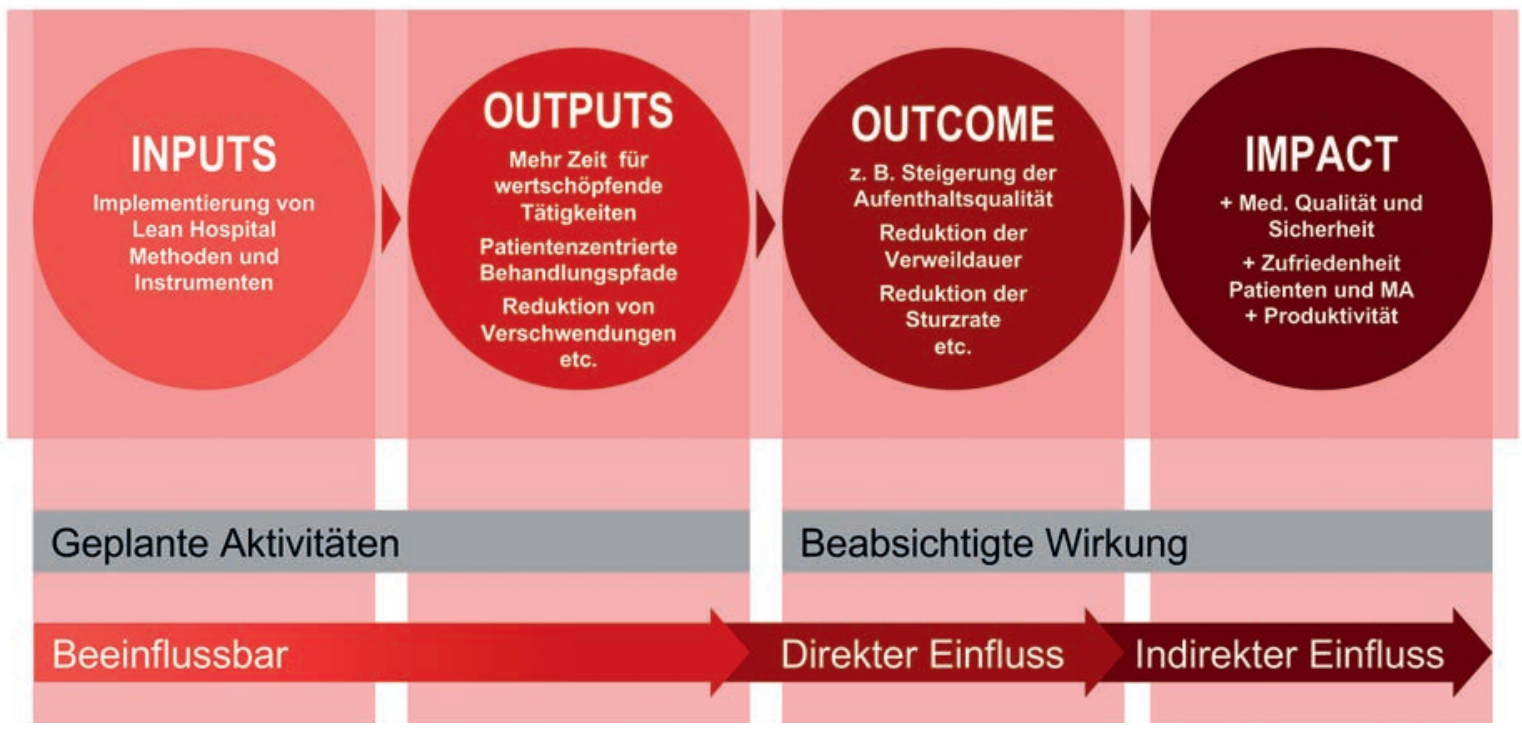

Abbildung 1: USB Wirkungsmodell Lean Hospital Management.

den Kennzahlen entwickelt, um einerseits Wirkungen zu erkennen und zu messen und andererseits den Stationsleitungen eine bessere Steuerung zu ermöglichen.

Die mit Lean Hospital eingesetzten Methoden und Instrumente (Inputs) erzielen Wirkungen. Diese werden sichtbar u.a. durch mehr Zeit für wertschöpfende Tätigkeiten, patientenzentrierte Behandlungspfade und die Reduktion von Verschwendungen (Outputs). Dies kann u.a. die Aufenthaltsqualität verbessern, die Aufenthaltsdauer verkürzen, aber auch die Stressbelastung des Personals reduzieren (Outcome). Letztlich sollte sich dies positiv auswirken auf die Patientenund Mitarbeiterzufriedenheit, die medizinische Qualität und Sicherheit sowie die Produktivität (Impact). Während die Input- und Output-Ebenen durch geplante Aktivitäten direkt beeinflussbar sind, sind die beabsichtigten Wirkungen auf der Outcome- und Impact-Ebene über einen direkten und/oder indirekten Einfluss erreichbar. Das aus diesen Wirkungsannahmen entstehende Modell versucht, die wichtigsten Einflussfaktoren und vermuteten Zusammenhänge transparent und somit diskutier- und überprüfbar darzustellen. Es gibt nur selten lineare Zusammenhänge der Wirkungsstufen.

Beispiel: Eine verbesserte Tagesplanung auf der Basis eines guten Überblicks (Input) führt u.a. zu einer Glättung der Arbeitslast (Output). Dies spürt der Patient mehr oder weniger direkt durch die Verminderung von Stress und Hektik und man kann annehmen, dass sich dies positiv auswirkt auf die Aufenthaltsqualität (Outcome), was wiederum die Patienten- und Mitarbeiterzufriedenheit steigert (Impact). Die bessere Tages- planung durch die Nutzung von interdisziplinären Kurztreffen sollte auch den Informationsfluss verbessern (Output). Dadurch können Patienten im Mittel früher entlassen werden (Outcome), was wiederum die Patientenzufriedenheit und Produktivität positiv beeinflussen kann (Impact).

\section{Kennzahlen-Modell einer Lean Bettenstation}

Geheimnisse lassen sich nicht managen. Man muss das Richtige sehen, um steuern und die Performance bewerten zu können. Hier ist das Wirkungsmodell ebenfalls hilfreich, da es Hinweise liefert, wo idealerweise gemessen werden müsste. Die Pragmatik kommt dann noch früh genug, da immer wieder der Messaufwand einschränkend wirkt. Daher seien ein paar grundsätzliche Prinzipien der Kennzahlenentwicklung und -beurteilung vorangeschickt.

1. Kennzahlen lösen taktisches Verhalten aus. Mitarbeitende tendieren dazu, Kennzahlen vorteilhaft darzustellen und wenn möglich zu beeinflussen, vor allem dann, wenn sie daran gemessen werden. Eine gute Kennzahl lenkt das (taktische) Verhalten der Mitarbeitenden in Richtung der Unternehmensziele.

2. Kennzahlen müssen fair sein. Mitarbeitende dürfen nur an dem gemessen werden, was sie mit ihrer Arbeit und dem Verhalten beeinflussen können. Sonst wirken Kennzahlen frustrierend.

3. Die Kausalitäts- bzw. Korrelationsannahmen müssen transparent sein. Dies ist einer der wichtigen Gründe für die Erarbeitung eines Wirkungsmodells. 
4. Die Kennzahlen müssen pragmatisch und messbar sein. Es gilt die Regel: Klein anfangen, praktikabel bleiben und das Messen weiterer, wichtiger Kennzahlen schrittweise aufbauen.

Um die Wirkungsannahmen zu überprüfen und die wesentlichen, systembestimmenden Elemente im Wirkungsmodell zu gewinnen, wurden zu den OutputOutcome-Beziehungen sowie den Outcome-ImpactBeziehungen Wirkungsmatrizen erstellt. Mit diesem Verfahren schätzten Stationsleiter und Mitglieder des Lean Programms die Wirkungsbeziehungen ein. Aus dieser Einschätzung konnten die kritischen Elemente in ein Wirkungssystem abgeleitet und die Zahl der notwendigen Kennzahlen systematisch reduziert werden [8]. Das verbleibende Set an Kennzahlen wurde daraufhin bewertet nach Fairness (Können die Mitarbeitenden die Kennzahl beeinflussen?), Messbarkeit (Was wird bereits gemessen? Wie gross ist der Messaufwand?), Steuerungsrelevanz (Sehen wir mit der Kennzahl, was wir steuern wollen, oder steuern wir nur, was wir sehen?) und taktisches Verhalten (Wie beeinflusst die Kennzahl das Verhalten der Mitarbeitenden?). Die Glättung der Arbeitslast kann z.B. durch die Varianz in der Leistungserfassung gemessen werden. Die Fairness und Steuerbarkeit sind dabei hoch, der Messaufwand klein und das taktische Verhalten gut.

\section{Das Identifizieren von Lean Effekten}

Eine wichtige Kennzahl ist die Aufenthaltsdauer des Patienten im Spital (Verweildauer), die auf verschiedene Art und Weise beeinflusst werden kann. Bei unserer Auswertung sind wir auf drei Ursachen gestossen, die eine Reduktion der Verweildauer bewirkt haben: Die Initiative eines Chefarztes, die Einführung des ERAS-Protokolls (Enhanced Recovery After Surgery) sowie die Einführung von Lean Hospital Management.

\section{Verbessertes Austrittsmanagement}

Auf einer Bettenstation verkürzte sich die Verweildauer (Abstand zum Mittelwert der Schweiz) bei konstantem CMI von 2,0 Tage (2014) und 1,9 Tage (2015) auf 1,2 Tage (2016) und schliesslich 0,5 Tage (2017). Auf Nachfrage, woher dieser Erfolg rühren könnte, stellte sich heraus, dass dieser im Wesentlichen durch ein aktives Austrittsmanagement des Chefarztes entsteht. Dadurch erhöhte sich die mittlere Fallzahl dieser Station pro Monat von 74 auf 84 , was eine Produktivitätssteigerung von $13,5 \%$ bedeutet. Hier zeigt sich, dass die Messung einer Kennzahl Fragen erzeugen kann.

\section{Effekte des ERAS-Protokolls}

In einem anderen Fachgebiet wurde bei zwei Patientengruppen das ERAS-Protokoll eingeführt, das mit einer ausführlichen Beschreibung des Prozesses für alle Beteiligten einschliesslich dem Patienten dafür sorgt, dass die Vorbereitung auf die OP (Ernährung, physiologischer Zustand) deutlich besser ist. Der Patient wird von einer eigenen Pflegefachkraft betreut und begleitet. Dank dem Protokoll befinden sich die Patienten postoperativ in einem deutlich besseren Allgemeinzustand. Das wiederum verkürzt die Verweildauer von im Mittel ca. 12 auf 6 Tage. Obwohl nur ein kleiner Teil der Patienten der entsprechenden Bettenstation anhand dieses Protokolls behandelt wurde, ist der Impact bei jedem einzelnen Patienten in den Mittelwerten der Station erkennbar. Das ERAS-Protokoll stellt den Patienten und sein Wohlergehen in den Mittelpunkt und richtet daran alle Massnahmen aus. Verschwendungen werden vermieden und das Protokoll laufend verbessert. Damit erfüllt es alle Lean Hospital Prinzipien [2] und ist - obwohl nicht Teil des Lean Programms - ein gutes Beispiel von gelebtem Lean Hospital (vgl. Tab. 1).

Tabelle 1: Erfüllung der Lean Hospital Prinzipien durch das ERAS-Protokoll.

\begin{tabular}{|c|c|c|}
\hline Lean Prinzip & Allgemeines Verständnis & Implizite Umsetzung im ERAS-Protokoll \\
\hline Wert & $\begin{array}{l}\text { Der Mehrwert für den Patienten steht im Mittel- } \\
\text { punkt aller Aktivitäten. }\end{array}$ & $\begin{array}{l}\text { Mit dem Fokus auf die optimale Fitness in der OP-Vor- und Nachbereitung wird dies } \\
\text { perfekt umgesetzt. Dem Patienten geht es deutlich besser und er ist früher wieder zu } \\
\text { Hause. }\end{array}$ \\
\hline Wertstrom & $\begin{array}{l}\text { Der Behandlungsprozess enthält für den Patienten } \\
\text { nur wertschöpfende Tätigkeiten. Verschwendung } \\
\text { ist eliminiert. }\end{array}$ & $\begin{array}{l}\text { Durch die Erarbeitung eines umfangreichen Leitfadens (Protokoll) und die kontinuier- } \\
\text { liche Begleitung durch eine ERAS-Pflegefachkraft werden nur direkte und indirekte } \\
\text { wertschöpfende Tätigkeiten ausgeführt, Verschwendung wird weitgehend vermie- } \\
\text { den. }\end{array}$ \\
\hline Fluss & $\begin{array}{l}\text { Es gibt keine Doppelarbeiten, Schleifen, Wartezei- } \\
\text { ten oder andere Umwege innerhalb des Prozesses. }\end{array}$ & $\begin{array}{l}\text { Durch klar definierte Rollen und die Abarbeitung eines definierten Protokolls wird ein } \\
\text { konsequenter Fluss der Tätigkeiten am Patienten hergestellt. }\end{array}$ \\
\hline Pull & $\begin{array}{l}\text { Produkte oder Dienstleistungen werden erst dann } \\
\text { hergestellt, wenn es einen Auftrag gibt. }\end{array}$ & $\begin{array}{l}\text { Dieses Prinzip ist bei Behandlungsprozessen naturgemäss erfüllt. Es erfolgt erst eine } \\
\text { Therapie, wenn eine Diagnose existiert. }\end{array}$ \\
\hline Perfektion & $\begin{array}{l}\text { Innerhalb eines kontinuierlichen Verbesserungs- } \\
\text { prozesses wird der Ablauf stetig verbessert }\end{array}$ & $\begin{array}{l}\text { Das Protokoll ist Gegenstand laufender Verbesserungen. Damit wird sichergestellt, } \\
\text { dass aktuelle Entwicklungen, Innovationen und Bedürfnisse ausgeführt werden. }\end{array}$ \\
\hline
\end{tabular}


Eine Ertragssteigerung von ca. CHF 4000 pro Fall [9] verdeutlicht zudem, dass eine Verbesserung der Qualität im Behandlungsprozess einen deutlich positiven betriebswirtschaftlichen Effekt auslöst. Es ist auch die Bestätigung des Produktivitäts-Paradoxes: «Wenn niedrige Kosten das Ziel sind, geht häufig die Qualität verloren. Wenn aber Qualität das Ziel ist, sinken in der Regel die Kosten» [10].

\section{Effekte von Lean Hospital}

Auch das Lean Programm zielt über verschiedene Instrumente auf eine Verkürzung der Verweildauer. Im Kern umgesetzt werden ein optimiertes und übersichtlicheres Tagesmanagement, eine höhere Frequenz in der Betreuung der Patienten, ein kontinuierlicher Verbesserungsprozess sowie eine verstärkte interprofessionelle und interdisziplinäre Zusammenarbeit zwischen Pflegenden und Ärzten. Eine Bettenstation des USB setzt diese Instrumente seit Ende 2014 ein. Im Vergleich zum Schweizer Mittelwert sank die Verweildauer um 1,1 Tage. Gleichzeitig sank der CMI nur um 0,1 und der Reduktions-Effekt lässt sich in allen drei auf dieser Station beteiligten medizinischen Fachgebieten beobachten. Hier scheint die Umsetzung von Lean Hospital erkennbar zu sein. Eine weitere Station führte Lean Hospital Anfang 2016 ein. Hier entwickelte sich der Abstand der Verweildauer zum Schweizer Mittel wie folgt: vor Einführung war die Station 1,5 Tage schlechter als der Mittelwert, nach Einführung verbesserte sich dieser Wert auf 0,2 über dem Mittelwert (2016, 2017). Dieser Effekt trat wiederum bei konstantem CMI auf und war in beiden dort beteiligten Fachrichtungen erkennbar (vgl. Abb. 2).

Tel +41613287489

katharina.ruether[at]usb.ch

\section{Zuordnung von Wirkungen in komplexen Systemen}

Eine klare und eindeutige Ursache-Wirkungs-Beziehung lässt sich in komplexen Systemen nicht aufzeigen. Messen können wir nur Korrelationen, die auch zufällig sein können. Je mehr aber Messwerte sichtbar gemacht und untereinander verglichen werden, desto eher lassen sich Wirkungen und ihre möglichen $\mathrm{Zu}$ sammenhänge erkennen und schätzen. Imperfekte Daten sind besser als keine, denn sie spornen das Lernen und Verbessern an [11]. Für ein Stations-Cockpit soll eine Sicht entwickelt werden, welche den Zustand und die Entwicklung einer Bettenstation auf einen Blick erkennen lässt. Eine solche dauerhafte 'Evaluation' der Performance durch die Stationsleitung dürfte auch die Steuerung vereinfachen und verbessern.

Soll ein positiver Effekt aber eindeutig einem Lean Projekt zugeordnet werden können und sind Aussagen über Ursache-Wirkungs-Beziehungen notwendig, so sind Verfahren der analytischen Statistik notwendig. Diese bedingen aber eine Reihe von Kennzahlen über einen grösseren Zeitraum (Pfadanalyse, Regressionsanalyse, Multi-Faktoren-Analyse). Das Wirkungsmodell kann als Ausgangspunkt dienen. Deshalb plant das USB das Wirkungs- und Kennzahlen-Modell zu validieren.

\section{Disclosure statement}

Es bestehen keine Interessenkonflikte.

\section{Bildnachweis}

Eigene Darstellung der Autoren.

Literatur

- Modig N, Åhlström P. Das ist Lean. Rheologica Publishing; 2015.

- Womack, JP, Jones, DT. Lean Thinking: Ballast abwerfen, Unternehmensgewinn steigern. 3. Aufl. Frankfurt am Main: Campus Verlag; 2013.

- Kenney C. Transforming Health Care: Virginia Mason Medical Center's Pursuit of the Perfect Patient Experience. NY: Productivity Press; 2011.

- Rossi, PH, Lipsey MW, Freeman HE. Evaluation: A Systematic Approach. Newbury Park: SAGE Publications, Inc.; 2003.

- Hofmann, J. Implizite Theorien in der Politik: Interpretationsprobleme regionaler Technologiepolitik. In Studien zur Sozialwissenschaft. Wiesbaden: VS Verlag für Sozialwissenschaften; 1993;Bd.132.

Walker D, Thierstein A, Betz P. Mythen und Märchen in der Politik: Vom Eigenleben politischer Annahmen. Verlag Rüegger; 1999.

- Khandker SR, Koolwal GB, Samad HA. Handbook on Impact Evaluation: Quantitative Methods and Practices. Washington: The World Bank; 2010.

- Vester F. Die Kunst, vernetzt zu denken. Ideen und Werkzeuge für einen neuen Umgang mit Komplexität. 6. Aufl. Stuttgart: dtv Wissen; 2007.

Behrendt $\mathrm{H}$. Evaluation Einsatz von ERAS. USB: Interner Bericht, 2017

- Skinner W. The Productivity Paradox. Management Review. 1986;75(9):41-45.

- Porter ME, Teisberg EO. Redefining Health Care. Boston: Harvard Business School Press; 2006. 